\title{
Total Synthesis of Ramonanins A-D**
}

\author{
Ross S. Harvey, Emily G. Mackay, Lukas Roger, Michael N. Paddon-Row,* \\ Michael S. Sherburn,* and Andrew L. Lawrence*
}

\begin{abstract}
The first total synthesis of the ramonanin family of lignan natural products is described. The short synthesis involves a 2,5-diaryl-3,4-dimethylene tetrahydrofuran intermediate, which participates in an unexpectedly facile DielsAlder dimerization, generating all four natural products. Insights into the reactivity and stereoselectivity of the key dimerization are provided through computational studies employing B3LYP/6-31G(d) and M06-2X/6-31G(d) model chemistries.
\end{abstract}

Guaiacum officinale and Guaiacum sanctum are the sources of the highly prized lignum vitae wood, known for its extraordinary strength and durability. The resin of lignum vitae, known as gum guaiac, or guaiac resin, is also valuable. It is reported to possess various medicinal properties, ${ }^{[1]}$ and has also found application in colorimetric tests for oxidative conditions (e.g., Nobles' test, ${ }^{[2]}$ Guaiac occult blood test, ${ }^{[3]}$ and Schönbein-Pagenstecher test $\left.{ }^{[4]}\right) . \alpha$-Guaiaconic acid (1) is the constituent of gum guaiac that is readily oxidized, forming the vividly colored guaiacum blue (2; Scheme $1 \mathrm{a}) .{ }^{[5]}$ Sadly, the highly sought-after attributes of lignum vitae wood and gum guaiac have led to overexploitation of Guaiacum officinale, which is now listed as an endangered species on the Red List of the International Union for the Conservation of Nature. ${ }^{[6]}$

The ramonanins A-D (3-6) are a group of spirocyclic phenylpropanoid tetramers isolated from the heartwood of Guaiacum officinale by Schroeder and co-workers in 2011 (Scheme $1 b){ }^{[7]}$ The ramonanins $(\mathbf{3}-\mathbf{6})$ formulate as dimers of $\alpha$-guaiaconic acid (1) and are reported to exhibit cytotoxic

[*] R. S. Harvey, E. G. Mackay, L. Roger, Prof. M. S. Sherburn Research School of Chemistry, Australian National University Canberra, ACT 2601 (Australia)

E-mail: michael.sherburn@anu.edu.au

Prof. M. N. Paddon-Row

School of Chemistry, The University of New South Wales

Sydney, NSW 2052 (Australia)

E-mail:m.paddonrow@unsw.edu.au

Dr. A. L. Lawrence

School of Chemistry, The University of Edinburgh

Joseph Black Building, West Mains Road, Edinburgh, EH9 3JJ (UK)

E-mail: a.lawrence@ed.ac.uk

[**] This work was supported by the Australian Research Council and a Marie Curie Career Integration Grant. M.N.P.-R. acknowledges that this research was undertaken with the assistance of resources provided at the $\mathrm{NCl}$ National Facility through the National Computational Merit Allocation Scheme supported by the Australian Government. The authors thank Prof. Schroeder (Cornell University) for kindly supplying samples of the ramonanin natural products and Tony Herlt (Australian National University) for assistance with HPLC separations.

orting information for this article is available on the WWW under http://dx.doi.org/10.1002/anie.201409818.

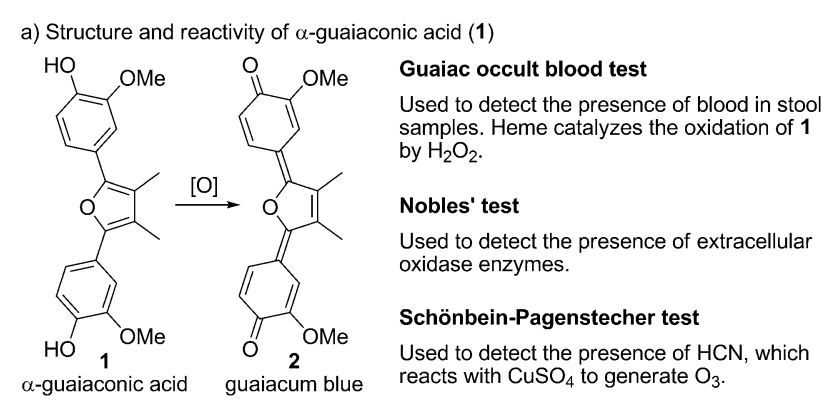

b) Schroeder's proposed biosynthesis of the ramonanins A-D (3-6)

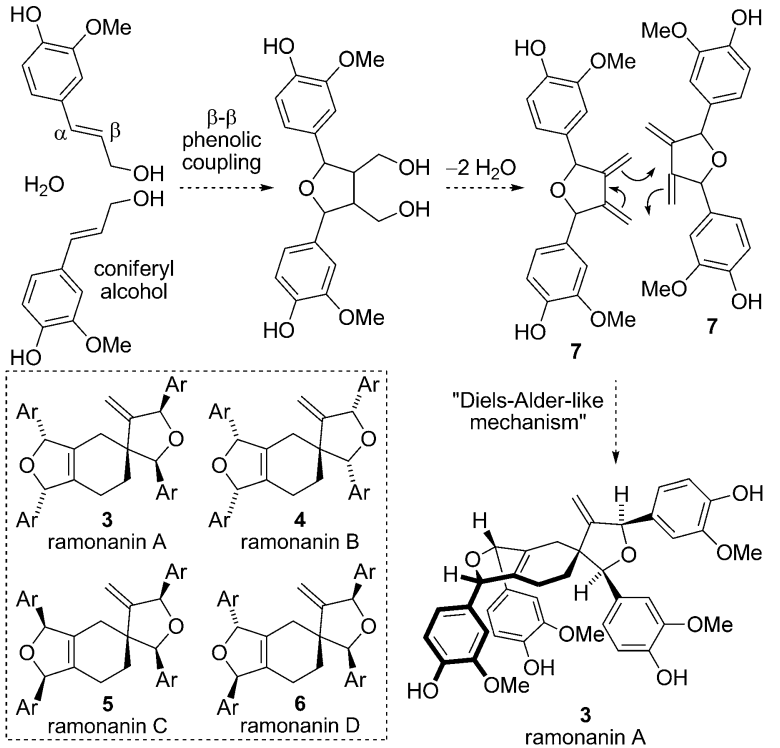

Scheme 1. a) The structure and reactivity of $\alpha$-guaiaconic acid (1). b) Schroeder's proposed biosynthesis of the ramonanins A-D (3-6). ${ }^{[7]}$

activity against human breast cancer cell lines. ${ }^{[7]}$ The endangered status of Guaiacum officinale, together with the promising biological activity of its natural products, are classic motivations for total synthesis efforts. ${ }^{[8]}$ In truth, however, our attention was drawn to these molecules because of their intriguing structures, which are fascinating from both a topological and biosynthetic perspective. The spirocyclic core of the ramonanins is unprecedented among natural products and Schroeder and co-workers proposed a biosynthetic pathway that involved dimerization of a lignan precursor, 7, through a "Diels-Alder-like mechanism" (Scheme $1 \mathrm{~b}) \cdot{ }^{[7]}$

Schroeder's proposed biosynthetic intermediate 7 presumably also serves as the biosynthetic precursor to $\alpha$ guaiaconic acid (1). There are no reports of 1,2-dimethylenecyclopentane-type structures undergoing Diels-Alder dimerizations under ambient conditions. ${ }^{[9]}$ It is, therefore, reason- 

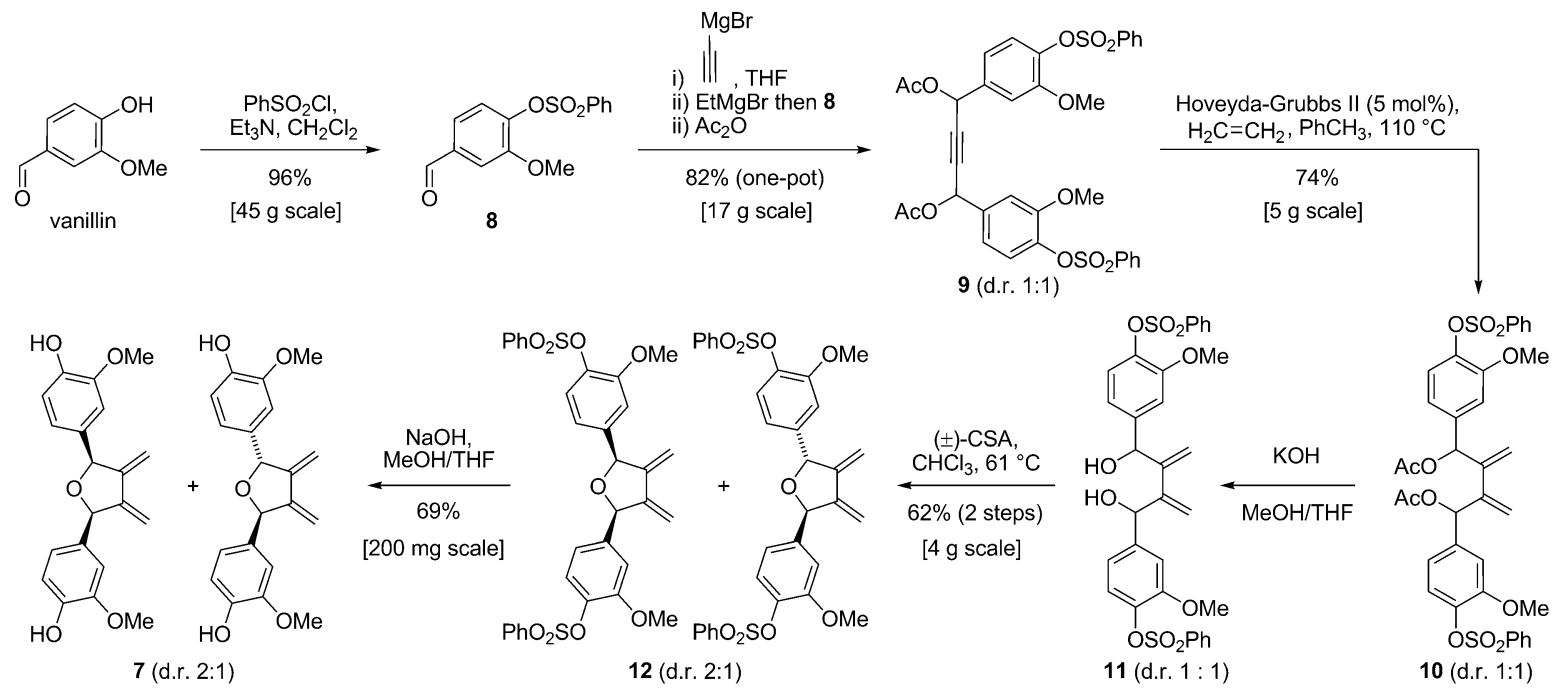

Scheme 2. Synthesis of lignan 7.

able to assume that lignan 7 would not undergo a spontaneous Diels-Alder dimerization. ${ }^{[10]}$ Our previous studies on the kingianin natural products utilized a radical-cation-catalyzed formal Diels-Alder (RCDA) reaction to dimerize a thermally unreactive bicyclo[4.2.0] octadiene structure. ${ }^{[1]}$ We considered that the ramonanins A-D (3-6) might also be the result of a biosynthetic RCDA dimerization. ${ }^{[12]}$ To investigate the nature of this fascinating biosynthetic dimerization we chose to pursue a total synthesis of the ramonanin natural products (3-6). ${ }^{[13]}$

The synthesis began with the high yielding conversion of vanillin into its benzene sulfonate ester, a process that could be easily conducted on large scale (Scheme 2). Two molecules of the resulting aldehyde $\mathbf{8}$ were united through an acetylene unit in a one-pot sequence to afford multigram quantities of diacetate 9 as a 1:1 mixture of diastereomers. Following a strategy pioneered by Mori and co-workers, ${ }^{[14]}$ alkyne 9 was subjected to an enyne metathesis with ethylene using the Hoveyda-Grubbs II catalyst, to afford diene $\mathbf{1 0}$ on multigram scale. Deprotection of the secondary alcohols was accomplished using $\mathrm{KOH}$ in $\mathrm{MeOH}$ to afford diol 11. Camphor sulfonic acid was then used to promote 3,4-dimethylene tetrahydrofuran ring formation, which generated protected lignan $\mathbf{1 2}$ as a 2:1 mixture of diastereomers, both of which would be required for the synthesis of ramonanins A-D (3-6). This short synthetic sequence allowed access to multigram quantities of key intermediate 12, which could be stored at $-15^{\circ} \mathrm{C}$ for weeks without deterioration. When required, the benzene sulfonate protecting groups could be cleanly removed with $\mathrm{NaOH}$ in $\mathrm{MeOH} / \mathrm{THF}$ to afford lignan 7 (Scheme 2).

To our surprise and delight, dimerization of lignan 7 was observed to occur spontaneously at room temperature, albeit slowly. ${ }^{[15]}$ Under optimized conditions, dissolution of the monomer cis- $^{7^{16]}}$ in a minimal amount of DMF and warming at $50^{\circ} \mathrm{C}$ provided, after chromatographic purification, a 54:9:37 mixture of ramonanins A (3), B (4), and C (5) in a combined yield of $45 \%$, in addition to $24 \%$ recovered starting material cis-7 (Scheme 3). ${ }^{[17]}$ Preparative HPLC

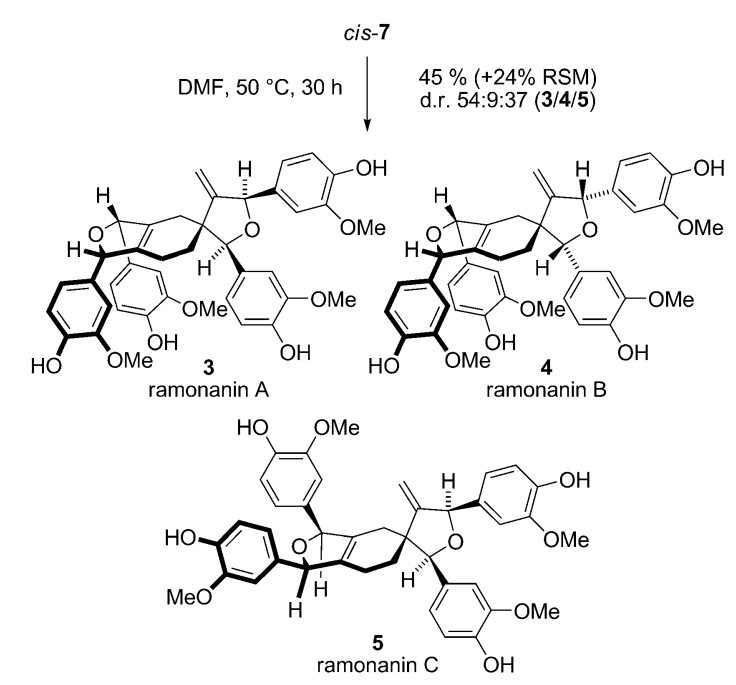

Scheme 3. Dimerization of lignan cis-7.

allowed the isolation of analytically pure samples of ramonanins A-C (3-5) and the spectroscopic data for each synthetic compound matched those reported for the corresponding natural product, thus securing the total synthesis of ramonanins A-C (3-5). Optical rotation values for the natural products were reported in the isolation paper $\left[3:[\alpha]_{\mathrm{D}}{ }^{22}-2.2\right.$ $(c 0.6, \mathrm{MeOH}) ; \mathbf{4}:[\alpha]_{\mathrm{D}}{ }^{22}-4.0(c 0.2, \mathrm{MeOH}) ; \mathbf{5}:[\alpha]_{\mathrm{D}}{ }^{22}-5.2(c$ $0.2, \mathrm{MeOH}) ; 6$ : $\left.[\alpha]_{\mathrm{D}}{ }^{22}-5.1(c 0.3, \mathrm{MeOH})\right] .^{[7]}$ Nevertheless, since ramonanins A-C are the Diels-Alder dimers of the achiral diene cis-7, we strongly suspected that these natural products were racemates. To test this hypothesis, we performed chiral HPLC analysis on samples of ramonanins A and $\mathrm{C}$, kindly provided by Professor Schroeder, ${ }^{[18]}$ which confirmed their racemic nature.

Our attention now turned to the final member of the family: ramonanin D (6; Scheme 1). A unique stereochemical feature of ramonanin $\mathrm{D}(\mathbf{6})$ is the trans relationship between the two aryl substituents on the dihydrofuran ring. As would 

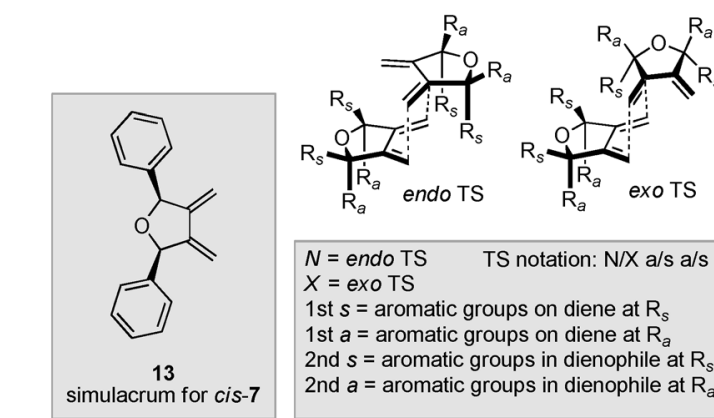

$N=$ endo $\mathrm{TS} \quad$ TS notation: $\mathrm{N} / \mathrm{X}$ a/s a/s

$X=$ exo TS

1 st $s=$ aromatic groups on diene at $R$

1 st $a=$ aromatic groups on diene at $R_{a}$

2nd $s=$ aromatic groups in dienophile at $R_{s}$

2nd $a=$ aromatic groups in dienophile at $R_{a}$
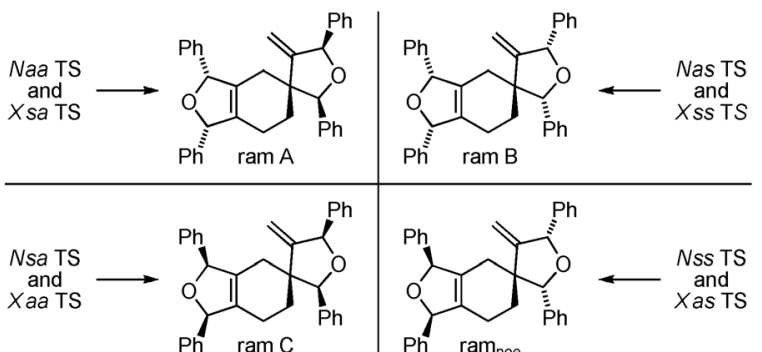

Scheme 4. The eight stereoisomeric TSs for the dimerization of 13 to afford the ramonanin simulacra ( $\operatorname{ram} A, \operatorname{ram} B, \operatorname{ram} C$, and $\operatorname{ram}_{\text {noe }}$ ), with explanation of the TS notation.

be expected, upon warming a mixture of cis-7 and trans-7, complex mixtures of products were obtained. Nevertheless, an analytically pure sample of ramonanin D (6) was isolated, albeit in low $(<1 \%)$ yield, after multiple HPLC purification steps.

Given the surprisingly facile nature of the Diels-Alder dimerization of cis-7, we chose to investigate the reactivity and stereochemical control of this process computationally, using the B3LYP/6-31G(d) and M06-2X/6-31G(d) model chemistries. The 2,5-diphenyl analogue of cis-7, namely $\mathbf{1 3}$ (Scheme 4), was used as a simulacrum. ${ }^{[19]}$ Eight distinct stereochemical modes of the Diels-Alder dimerization, and hence eight transition structures (TSs), are possible, and these are depicted in Scheme 4. The TSs are described using a notation, in which the Alder-Stein mode may be either endo $(N)$ or exo $(X)$ and the pair of phenyl substituents on the diene and on the dienophile may be either anti $(a)$ or syn $(s)$ with respect to the bond-forming zone. The eight TSs comprise four pairs, both members of each pair giving one of the ramonanin simulacra adducts ( $\operatorname{ram} \mathrm{X}=$ simulacra of ramonanin $\mathrm{X} ; \operatorname{ram}_{\text {noe }}=$ not observed experimentally).

The computed product distribution for the Diels-Alder dimerization of $\mathbf{1 3}$ is presented in Table 1, together with the experimentally determined product distribution from dimerization of cis-7 (Scheme 3). The agreement between the predicted and experimental adduct distribution is good and strongly suggests that not only is the model chemistry used in this study reliable, but also that the $\mathrm{OH}$ and $\mathrm{OMe}$ substituents in cis-7 do not significantly affect the distribution of the ramonanin stereoisomers. As expected, the Naa and Xaa TSs are the most favorable, presumably because both pairs of phenyl groups (i.e., from diene and dienophile) occupy the anti space with respect to the bond-forming zone, with the former lying $3 \mathrm{~kJ} \mathrm{~mol}^{-1}$ below the latter, in accordance with
Table 1: B3LYP/6-31G(d) and experimental product distributions [\%] for the Diels-Alder dimerization of 13 and cis-7, respectively.

\begin{tabular}{lllll}
\hline Adduct & Mode & Predicted & Total predicted & Experimental \\
\hline ram A & $\begin{array}{l}\text { Naa } \\
\text { Xsa }\end{array}$ & 58.6 & 59 & 54 \\
& & 0.1 & & \\
ram B & Nas & 3.7 & 4 & 9 \\
& Xss & 0 & & \\
& Nsa & 17.4 & 35 & 37 \\
$\operatorname{ram~C}$ & Xaa & 17.8 & & \\
& Nss & 0.1 & 2 & - \\
$\operatorname{ram}_{\text {noe }}$ & Xas & 2.3 & 2 & \\
\hline
\end{tabular}

Alder's endo rule. TSs with all phenyl groups positioned in the syn space, are 16 (Nss) and $24 \mathrm{~kJ} \mathrm{~mol}^{-1}$ (Xss) above the Naa $\mathrm{TS}$, reflecting steric congestion of the phenyl groups, as may be seen by comparing the geometries of Naa and Nss TSs in Figure 1. All TSs were found to be highly asynchronous, with the shorter of the newly formed bonds between 1.72 and $1.97 \AA$ and the longer one between 3.04 and $3.69 \AA$, the most asynchronous being the Nss TS $(\Delta r=1.97 \AA$, Figure 1$)$.
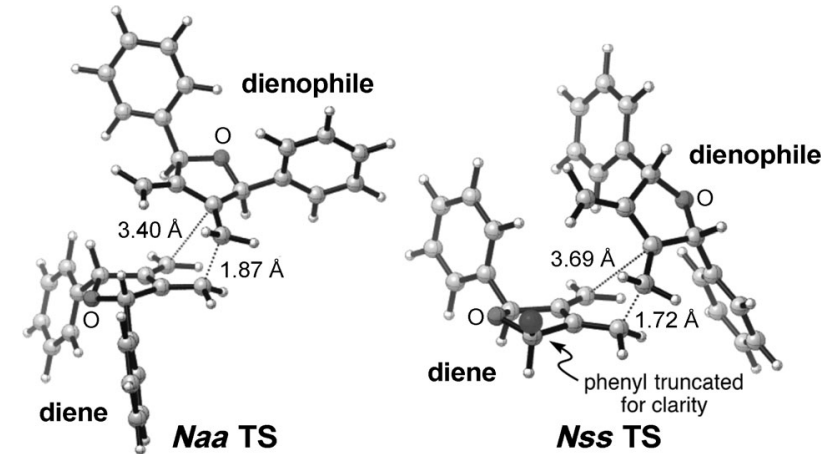

Figure 1. Transition Structures Naa and Nss with bond lengths of the newly formed bonds.

It seems surprising that neat samples of cis-7 readily dimerize, as does cyclopentadiene (CPD), given that the terminal methylene carbon atoms in $\mathbf{1 3}$ are calculated to be $3.139 \AA$ apart, compared to only $2.363 \AA$ in CPD ${ }^{[20]}$ The M06$2 \mathrm{X}$ computed activation enthalpies and free energies for the dimerization of CPD, 1,2-dimethylenecyclopentane, 3,4dimethylenetetrahydrofuran, and $\mathbf{1 3}$ are listed in Table 2.

As may be seen from Table $2, \Delta H^{+}$for Diels-Alder dimerization is approximately the same for all four reactants and, in particular, neither the presence of phenyl groups nor that of the ring oxygen has any significant effect on $\Delta H^{+}$ (compare entries 3 with 4 and 2 with 3 ). However, $\Delta G^{*}$ for the dimerization of $\mathbf{1 3}$ is about $10 \mathrm{~kJ} \mathrm{~mol}^{-1}$ higher than in the other three systems, for which similar values are obtained. This free energy difference translates into a 50-fold decrease in the Diels-Alder dimerization rate for 13, compared to the other dienes listed in Table 2. The origin of this effect is probably due to the phenyl groups in $\mathbf{1 3}$ experiencing a steeper potential for their torsional motion about the bonds connecting them to the tetrahydrofuran ring in the TS, compared to 
Table 2: $\Delta H^{+}$and $\Delta G^{\neq}$values $\left.(\mathrm{k}) \mathrm{mol}^{-1}, 25^{\circ} \mathrm{C}\right)$ for the Diels-Alder dimerization of various dienes. ${ }^{\text {[a] }}$

Entry

[a] M06-2X/6-31G(d)-[PCM = THf]//M06-2X/6-31G(d) activation energies from the lowest energy (endo) path.

the reactant. In conclusion, these calculations support the notion of a concerted cycloaddition event, both in the synthesis and biogenesis of the ramonanin natural products.

In summary, a seven-step total synthesis of the ramonanin natural products has been achieved. An investigation of the chemistry of Schroeder's proposed biosynthetic pathway has pinpointed the most likely origin of these fascinating structures. The natural products have been shown to be racemates. Our experimental results demonstrate that lignan $\mathbf{7}$ is predisposed toward thermal Diels-Alder dimerization, and our computational findings predict this behavior more widely in the 1,2-dimethylene cyclopentane series. It remains unclear whether this predisposed reactivity is being harnessed in nature constructively, or is generating products that are artifacts of isolation.

Received: October 6, 2014

Published online:

Keywords: biomimetic synthesis - Diels-Alder reactions . enyne metathesis · lignans $\cdot$ natural products

[1] a) R. S. Munger, J. Hist. Med. Allied Sci. 1949, IV, 196-229; b) J. F. Morton, Atlas of Medicinal Plants of Middle America: Bahamas to Yucatan, Charles C. Thomas, Springfield, 1981; c) J. L. Hartwell, Lloydia 1971, 34, 386-425; d) L. P. Somogyi, Food Additives, Wiley, New York, 2000.

[2] M. K. Nobles, Can. J. Bot. 1958, 36, 91-99.

[3] D. C. Rockey, N. Engl. J. Med. 1999, 341, 38-46.

[4] W. Autenrieth, Laboratory manual for the detection of poisons and powerful drugs, 4th ed. (Ed.: W. H. Warren), Blakiston, Philadelphia, 1915, pp. 21-22.

[5] The structure of guaiacum blue (2) in Scheme 1a is that shown in the following publication, but it should be noted that this compound has never been fully characterized, see: J. F. Kratochvil, R. H. Burris, M. K. Seikel, J. M. Harkin, Phytochemistry 1971, 10, 2529-2531.

[6] "Guaiacum officinale L." Taxonomic Serial No.: 897104 Retrieved [07-18-2014], from the ITIS (Integrated Taxonomic
Information System) on-line database, http://www.itis.gov. Listed as endangered on the IUCN (International Union for the Conservation of Nature) Red List. Retrieved [07-18-2014], from http://www.iucnredlist.org.

[7] K. J. Chavez, X. Feng, J. A. Flanders, E. Rodriguez, F. C. Schroeder, J. Nat. Prod. 2011, 74, 1293-1297.

[8] For a discussion of the role of total synthesis in addressing the problem of natural product supply, see J. D. Keasling, A. Mendoza, P. S. Baran, Nature 2012, 492, 188-189.

[9] This lack of literature precedent perhaps explains why Schroeder and co-workers described the key step in their proposed biosynthesis as having a "Diels-Alder-like mechanism" (Scheme 1). The most closely related example of this type of Diels - Alder reaction comes from Bloomquist and co-workers who, in 1956, synthesized 1,2-dimethylenecyclopentane and reported that attempts to distil the product at $97^{\circ} \mathrm{C}$ resulted in dimerization, see: A. T. Blomquist, J. Wolinsky, Y. C. Meinwald, D. T. Longone, J. Am. Chem. Soc. 1956, 78, 6057-6063.

[10] For an excellent review on biosynthetic Diels-Alder reactions, see: E. M. Stocking, R. M. Williams, Angew. Chem. Int. Ed. 2003, 42, 3078-3115; Angew. Chem. 2003, 115, 3186-3223.

[11] S. L. Drew, A. L. Lawrence, M. S. Sherburn, Angew. Chem. Int. Ed. 2013, 52, 4221-4224; Angew. Chem. 2013, 125, 4315-4318.

[12] Relatively few examples of total syntheses containing proposed biomimetic radical cation Diels-Alder reactions have been reported, see Ref. [11] and: a) H. Cong, C. F. Becker, S. J. Elliot, M. W. Grinstaff, J. A. Porco, J. Am. Chem. Soc. 2010, 132, 75147518; b) S. Lin, M. A. Ischay, C. G. Fry, T. P. Yoon, J. Am. Chem. Soc. 2011, 133, $19350-19353$; c) H. N. Lim, K. A. Parker, Org. Lett. 2013, 15, 398 -401; d) C. Qi, H. Cong, K. J. Cahill, P. Müller, R. P. Johnson, J. A. Porco, Angew. Chem. Int. Ed. 2013, 52, $8345-$ 8348; Angew. Chem. 2013, 125, 8503-8506; e) H. N. Lim, K. A. Parker, J. Org. Chem. 2014, 79, 919-926.

[13] For our previous biomimetic studies on dimeric natural products, see Ref. [11] and: a) P. D. Brown, A. C. Willis, M. S. Sherburn, A. L. Lawrence, Angew. Chem. Int. Ed. 2013, 52, 13273-13275; Angew. Chem. 2013, 125, 13515-13517; b) P. D. Brown, A. C. Willis, M. S. Sherburn, A. L. Lawrence, Org. Lett. 2012, 14, $4537-4539$.

[14] Selected examples: a) A. Kinoshita, N. Sakakibara, M. Mori, J. Am. Chem. Soc. 1997, 119, 12388-12389; b) K. Tonogaki, M. Mori, Tetrahedron Lett. 2002, 43, 2235-2238; c) M. Mori, K. Tonogaki, N. Nishiguchi, J. Org. Chem. 2002, 67, 224-226.

[15] The half-lives of both diastereomers of lignan 7 were determined to be approximately 2 days, for mixtures of diastereomers dissolved in a minimum of $\operatorname{DMF}(14.6 \mathrm{M})$ at $25^{\circ} \mathrm{C}$; see the Supporting Information for details.

[16] The cis and trans diastereomers of compound $\mathbf{1 2}$ could be separated by preparative HPLC and then used to prepare pure samples of cis-7 and trans-7, see the Supporting Information for details.

[17] The reaction was halted before completion to avoid the formation of decomposition products. Attempts to bring about a RCDA reaction using aminium radical catalysis (D. A. Bellville, D. D. Wirth, N. L. Bauld, J. Am. Chem. Soc. 1981, 103, 718720), or photoredox catalysis (Ref. [12b]), were met with failure.

[18] An authentic sample of ramonanin B was not available.

[19] Full details of these calculations are given in the Supporting Information.

[20] The fast Diels - Alder dimerization of cyclopentadiene has been ascribed to its short $\mathrm{C} 1-\mathrm{C} 4$ distance, see: R. Sustmann, M. Böhm, J. Sauer, Chem. Ber. 1979, 112, 883-889. 


\section{Communications}

Total Synthesis

R. S. Harvey, E. G. Mackay, L. Roger,

M. N. Paddon-Row,* M. S. Sherburn,*

A. L. Lawrence*

III-IIII

Total Synthesis of Ramonanins A-D

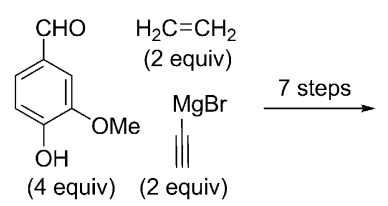

Dimers of dimers: The first total synthesis of the ramonanin family of natura products has been achieved in short order. These natural phenylpropanoid tetramers were assembled in seven steps

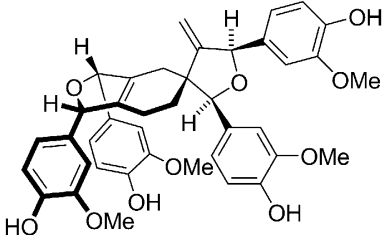

ramonanin $\mathrm{A}$

from the starting materials vanillin, ethylene, and the ethynyl Grignard reagent. Computational studies shed light on a surprisingly facile Diels-Alder dimerization. 